\title{
TRASTORNOS RESPIRATORIOS EN NIÑOS CON SÍNDROME DE DOWN
}

\author{
Respiratory disorders in children with down syndrome
}

Dra Carolina Campos

Pediatra Especialista en Enfermedades Respiratorias

Unidad de Broncopulmonar Infantil

Complejo Asistencial Dr Sótero del Río

\section{RESPIRATORY DISORDERS IN CHILDREN WITH DOWN SYNDROME}

Respiratory disorders in children with Down syndrome (DS) are common, and frequently undiagnosed. They often present sleep disordered breathing, respiratory tract infections and recurrent wheezing. Children with DS also have associated conditions that contribute to recurrent respiratory problems, such as hypotonia, immune disorders, congenital heart disease and gastroesophageal reflux. An adequate knowledge of all possible causes of respiratory pathology in children with DS is necessary, in order to allow a proper diagnosis, management and prevention of complications.

Key words: Down syndrome, sleep obstructive apnea, respiratory infections, asthma, respiratory aspiration.

\section{RESUMEN}

Los problemas respiratorios en niños con Síndrome de Down (SD) son frecuentes y muchos de ellos subdiagnosticados siendo los más habituales los trastornos respiratorios del sueño, infecciones respiratorias y sibilancias recurrentes. Los niños con SD, además, tienen condiciones asociadas que contribuyen a sus problemas respiratorios recurrentes, como hipotonía, alteraciones inmunes, cardiopatías congénitas y reflujo gastroesofágico. Se debe tener un adecuado conocimiento de todas las posibles causas de patología respiratoria en niños con SD, para hacer un adecuado diagnóstico, tratamiento y prevención de las complicaciones.

Palabras claves: síndrome de Down, síndrome de apnea obstructiva del sueño, infecciones respiratorias, asma, aspiración pulmonar.

\section{INTRODUCCIÓN}

El Síndrome de Down (SD), causado por trisomía del gen 21, es la anomalía cromosómica más frecuente, afectando a 1 de cada 600 a 800 recién nacidos vivos. Se caracteriza por rasgos físicos particulares (Figura 1), mayor riesgo de malformaciones congénitas, mayor vulnerabilidad para presentar enfermedades y discapacidad física e intelectual en grado variable $(1,2)$. En las últimas décadas la esperanza de vida de los niños con SD ha aumentado en forma significativa, principalmente por el tratamiento quirúrgico precoz de las cardiopatías congénitas y mejor tratamiento de las anomalías congénitas del tracto gastrointestinal $(1,2)$.

Los problemas respiratorios son una causa importante de morbilidad y mortalidad en estos niños, constituyendo el principal motivo de hospitalización. Presentan diversas condiciones alguno de los cuales pueden contribuir al desarrollo de enfermedades respiratorias, como hipotonía, anomalías craneofaciales, problemas cardíacos y gastrointestinales, retraso del desarrollo psicomotor (3-5).

\section{Correspondencia:}

Dra Carolina Campos

Unidad Broncopulmonar Infantil

Complejo Asistencial Dr. Sótero del Río

Av. Concha y Toro 3459, Puente Alto

Santiago, Chile.

Email: carolinacamposo@gmail.com
El objetivo de esta revisión es describir los problemas respiratorios en niños con SD, con el fin de aumentar su identificación, y de esta forma prevenir complicaciones, y obtener una mejor calidad de vida.

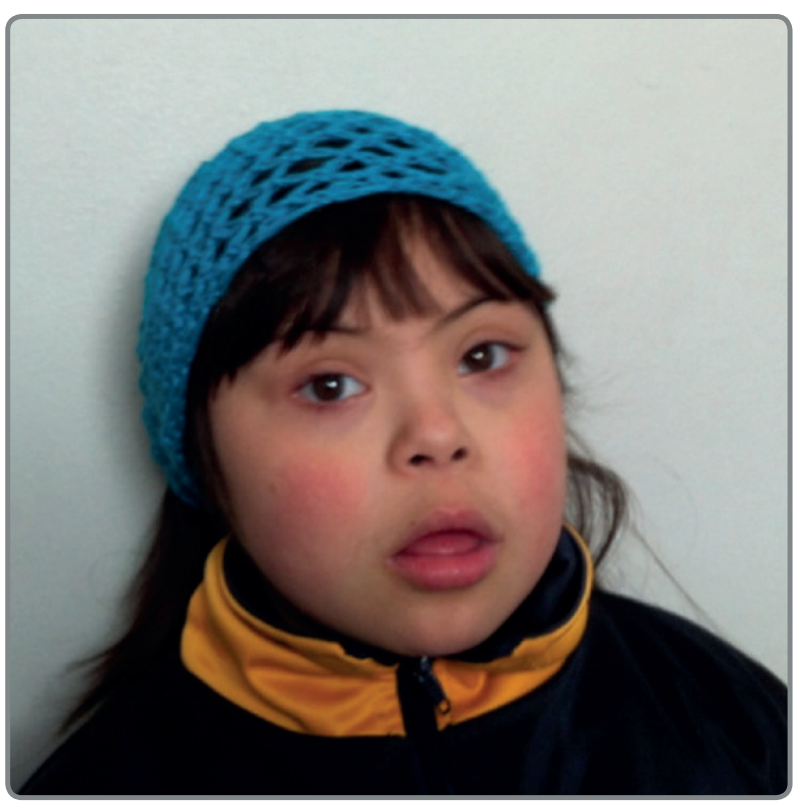

Figura 1.

Niña con Síndrome de Down. Observar puente nasal plano, macroglosia.

(Publicación de fotografía autorizada por tutor) 


\section{TRASTORNOS RESPIRATORIOS DEL SUEÑO}

Los trastornos respiratorios del sueño (TRS) son el problema respiratorio más frecuente. El síndrome de apnea obstructiva del sueño (SAOS) afecta a niños de cualquier edad, con una prevalencia de 50-75\% (dependiendo del instrumento utilizado en la investigación: saturometría nocturna, polisomnograma u otro), a diferencia de la prevalencia de $2 \%$ en la población pediátrica general (1-3). De los niños con SD que roncan, el 97\% presenta SAOS de grado variable (4-6). Las características anatómicas y dinámicas que contribuyen al desarrollo de SAOS son la hipoplasia medio facial y mandibular, vía aérea nasal estrecha, macroglosia relativa y glosoptosis, hipertrofia amigdaliana y adenoidea, malacia de vía aérea, obesidad, e hipotonía faríngea. Por esta última se produce un colapso de los músculos faringeos durante la inspiración.

Los TRS tienen una amplia gama de síntomas y signos, incluyendo ronquido, posiciones inhabituales al dormir, enuresis, cansancio, falta de crecimiento, trastorno del aprendizaje, falta de atención e inquietud psicomotora, falta de energía e iniciativa en actividades escolares y además hipertensión pulmonar (4). Se debe tener una alta sospecha en estos pacientes y no confundir Ios síntomas de un posible SAOS con características propias del SD. Existen estudios que muestran que los padres no detectan los problemas para dormir, al comparar sus reportes con polisomnografías $(3,6)$. La Academia Americana de Pediatría, en su guía de supervisión de salud para niños con SD (7) recomienda preguntar por síntomas de SAOS al menos una vez antes de los 6 meses y derivar si es necesario, después del año preguntar por síntomas en cada consulta, y realizar una polisomnografía a todos los niños con SD a los 4 años. Dado el alto costo de la polisomnografía una alternativa es el uso de poligrafía (Figura 2).

El tratamiento debe incluir a todos los posibles factores que contribuyen, bajar de peso en pacientes obesos, realizar adenoamigdalectomía, tratamiento de rinitis. En caso de persistir el SAOS puede ser necesario utilizar ventilación no invasiva nocturna. Se describe la utilización de aparatos dentales que mantienen permeable la vía aérea durante el sueño, y tratamiento quirúrgico $(8,9)$.

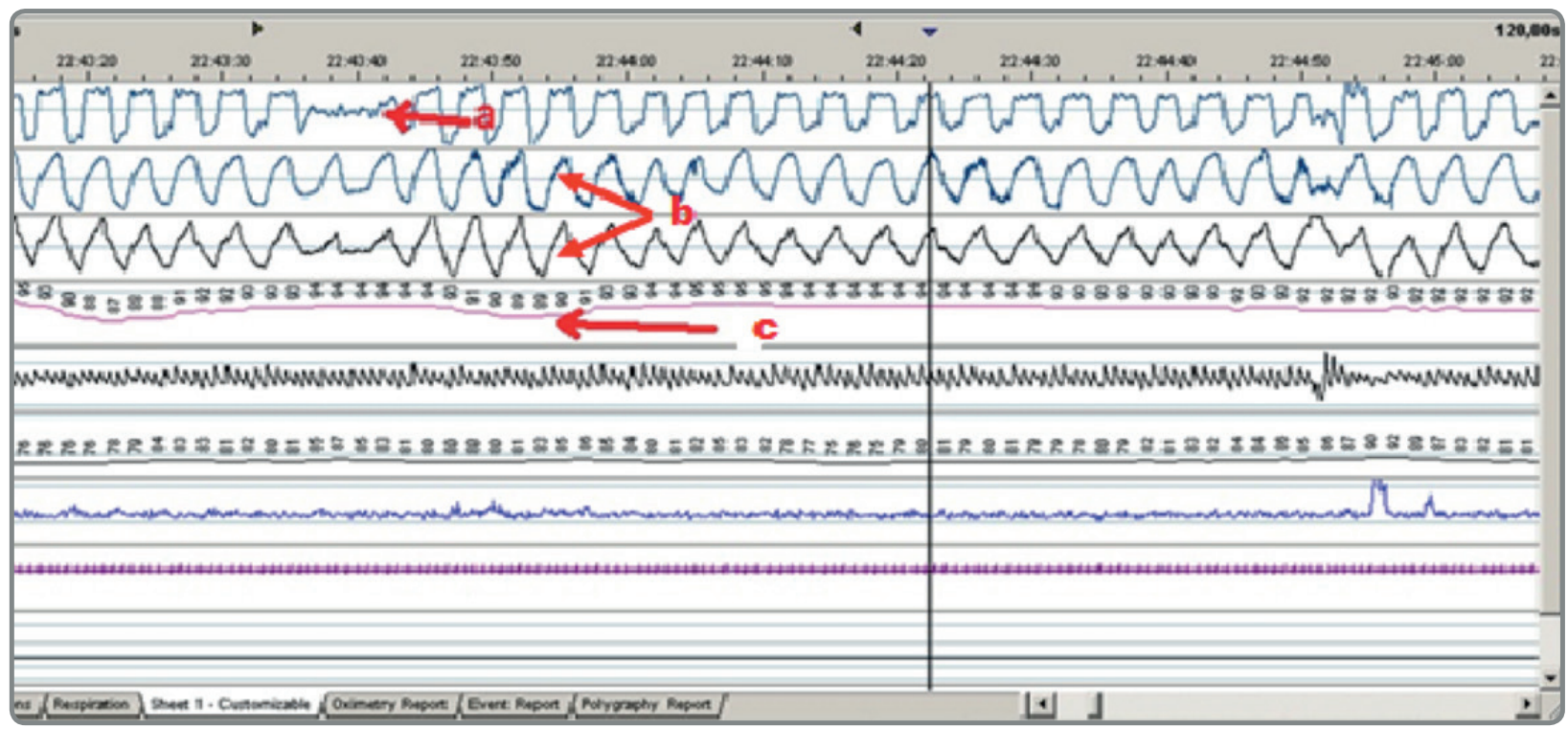

Figura 2.

Poligrafía que muestra apnea obstructiva en niña de 10 años con Síndrome de Down. (a) Ausencia de flujo nasal con (b) movimientos torácico y abdominal y (c) caída de saturación hasta 89\% después del evento.

\section{ALTERACIONES DE LA VÍA AÉREA}

Las alteraciones de la vía aérea son más frecuentes que en niños sin SD con síntomas respiratorios persistentes. Presentan alteraciones anatómicas y funcionales, tanto de la vía aérea superior como inferior (10).

Las alteraciones de la vía aérea superior fueron descritas en los TRS. En la Tabla 1 se describen las alteraciones endoscópicas encontradas en vía aérea inferior en 24 pacientes con SD que tenían síntomas respiratorios recurrentes, destacando que 11 tenían más de una alteración (10). En otras series, la laringomalacia (Figura 3) y traqueomalacia (Figura 4) fueron las más frecuentemente descritas $(10,11)$. La primera se presenta con estridor inspiratorio, la traqueomalacia con estridor inspiratorio o bifásico. La estenosis subglótica también ha sido descrita más frecuentemente en niños son SD (12). Dado la frecuente intubación de éstos pacientes (por cirugías cardíacas y digestivas), es posible que sea más frecuente la estenosis subglótica adquirida (4). Shott (13) estudió las dimensiones de la tráquea en niños con SD con resonancia 
nuclear magnética, encontrando que tienen una vía aérea más pequeña. Por esto recomienda utilizar tubo endotraqueal (TET) a lo menos 2 números menores para su edad o peso, con el fin de evitar el desarrollo de estenosis subglótica. Se describe también en niños con SD bronquiomalacia, estenosis traqueal, anillos traqueales y bronquio traqueal, este último se manifiesta con atelectasia y/o neumonía recurrente o persistente del lóbulo superior derecho (10).

Se debe sospechar la presencia de alteraciones de la vía aérea inferior en niños con SD que manifiestan tos crónica, sibilancias y respiración ruidosa, considerarlas dentro del diagnóstico diferencial de asma o infecciones recurrentes. La broncoscopía flexible es el procedimiento de elección para su diagnóstico.

\begin{tabular}{|l|c|}
\hline Laringomalacia & $50 \%$ \\
\hline Traqueomalacia & $33 \%$ \\
\hline Estenosis subglótica & $4 \%$ \\
\hline Broncomalacia & $21 \%$ \\
\hline Bronquio traqueal & $21 \%$ \\
\hline Estenosis traqueal & $4 \%$ \\
\hline Anillo traqueal & $4 \%$ \\
\hline
\end{tabular}

\section{Tabla 1.}

Alteraciones broncoscópicas de la vía aérea en niños con Síndrome de Down y síntomas respiratorios recurrentes (Autorizado por autor principal (10)).

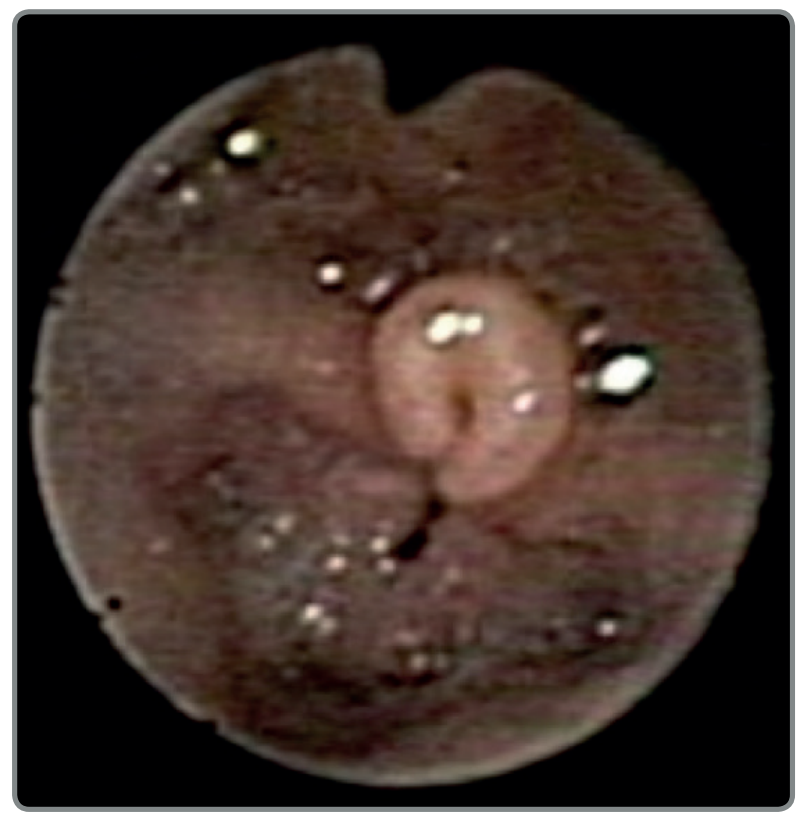

Figura 3.

Broncoscopía flexible: laringomalacia, epíglotis en omega y repliegues aritenoideos redundantes que se colapsan inspiración.

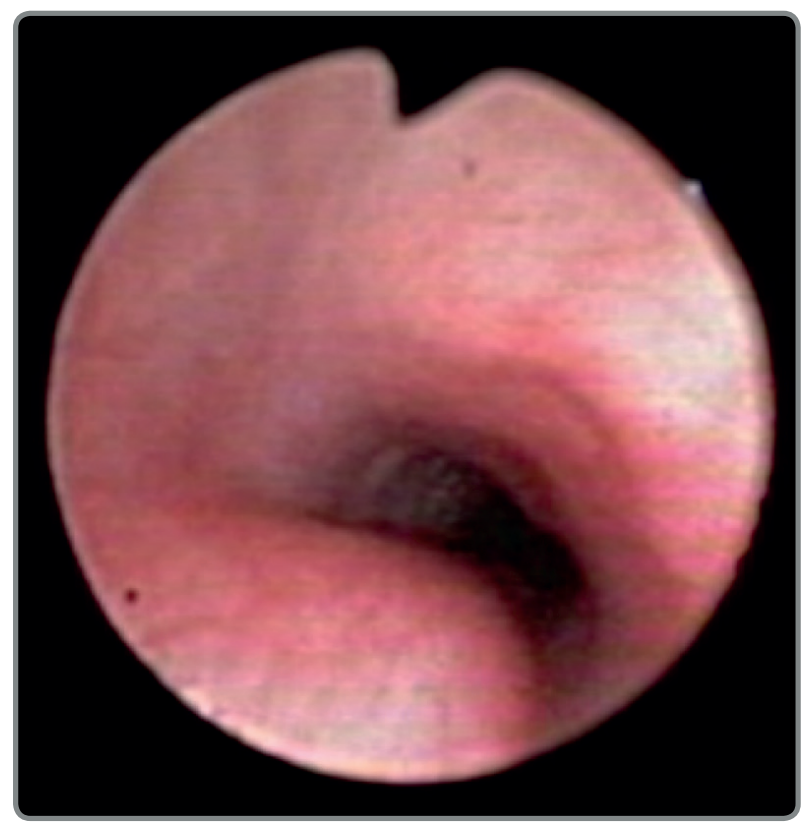

Figura 4.

Broncoscopía flexible: traqueomalacia proximal.

\section{SIBILANCIAS RECURRENTES Y ASMA}

Las sibilancias recurrentes son frecuentes en estos pacientes, presentándose en un tercio de ellos (14). Hilton mostró que los pacientes con SD reportaban mayor frecuencia de sibilancias los últimos 12 meses comparado con sus hermanos y con controles sanos, con un riesgo relativo (RR) de 2.8 y 2.7 respectivamente (15).

Estudios sugieren que pacientes con SD no tienen mayor prevalencia de asma que los niños sin SD, tienen menos enfermedad atópica y menor cantidad de test cutáneo positivo. Existen otros factores en estos pacientes que están asociados a mayor prevalencia de sibilancias recurrentes, como alteraciones pulmonares congénitas, cardiopatías congénitas, colapso de vía aérea superior por hipotonía, anomalías de vía aérea (traqueomalacia) y aspiración crónica. Estos últimos 2 factores deben ser buscados activamente (4).

\section{INFECCIONES RESPIRATORIAS Y COMPROMISO INMUNOLÓGICO}

Los niños con SD presentan mayor riesgo de tener infecciones respiratorias agudas (IRA) comparado con niños sin SD. Son la principal causa de hospitalización (50\%), tienen un curso más severo y presentan mayor mortalidad $(14,15)$. Las más frecuentes son neumonía y bronquiolitis, requieren más ingreso a unidad de paciente crítico, y el tiempo medio de estadía y los costos de la hospitalización son 2 a 3 veces mayores que en niños con el mismo diagnóstico sin SD (15). El virus respiratorio sincicial es una causa importante de IRA, 
requieren más hospitalización que niños sin SD, tienen un curso más severo y estadías hospitalarias más prolongadas (16). Se considera profilaxis con Palivizumab a todos los niños con SD, no solo quienes presentan cardiopatía congénitas(1).

La mayor incidencia de IRA en estos pacientes es multifactorial, juegan su rol alteraciones de la vía aérea ya descritas, reflujo gastroesofágico con aspiración pulmonar, defectos inmunológicos. Las alteraciones del sistema inmune incluyen: linfopenia de células T y B leve a moderada, niveles bajos de subclases de Inmunoglobulina $\mathrm{G}$, defecto en la quimiotaxis de neutrófilos, respuesta disminuida de anticuerpos específicos a las inmunizaciones, timo de tamaño más pequeño, disminución de Inmunoglobulina A total y específica en saliva (17).

Se recomienda vacunación contra influenza anual, vacuna antineumocócica polisacárida a niños con enfermedad cardíaca o pulmonar crónica desde los 2 años, una vigilancia aumentada de síntomas y signos de IRA y una precoz intervención en el curso de ésta (7).

\section{ENFERMEDADES DE LA VASCULATURA PULMONAR}

La vasculatura pulmonar en niños con SD es más vulnerable a presentar patología, con manifestaciones como hipertensión pulmonar (HTP), edema pulmonar o hemorragia pulmonar.

Los niños con SD y cardiopatía congénita son más susceptibles a presentar HTP, especialmente en presencia de defecto del septo atrioventricular, presentando HTP en forma precoz y de mayor severidad $(3,4)$. Además, presentan HTP más fácilmente al cursar enfermedad hipóxica, como es el caso de SAOS y daño pulmonar crónico. El tratamiento precoz de estas patologías evita la HTP. También tienen más frecuentemente hipertensión pulmonar persistente del recién nacido.

El edema pulmonar se desarrolla más frecuentemente en niños con SD. Se ha reportado edema pulmonar intra y postoperatorio en niños con SD con SAOS, en relación a la rápida desaparición de la obstrucción de vía aérea superior, principalmente posterior a amigdalectomía. Los niños con SD y cardiopatía congénita tendrían mayor probabilidad para desarrollar edema pulmonar de altura. Sin embargo la exposición a mayores alturas también podría determinar la aparición de HTP $(3,4)$.

La hemorragia pulmonar (HP) afecta más frecuentemente a niños con SD. Puede presentarse en forma aguda con hemoptisis o falla respiratoria; subaguda, con deficiencia de hierro, disnea crónica o enfermedad intersticial difusa; o asintomática, y ser un hallazgo en LBA realizado por otra razón. La HP puede presentarse en niños con SD con cardiopatías congénitas, neumonías recurrentes, aspiración pulmonar y malformaciones pulmonares. Fenómenos inmunes y enfermedad celíaca también han sido asociados a HP $(3,4)$.

\section{ENFERMEDADES DEL PARÉNQUIMA PULMONAR}

Se describen algunas alteraciones de la estructura pulmonar, pero su relación con la morbilidad respiratoria de estos pacientes no es clara. Los hallazgos histopatológicos incluyen aspecto poroso del pulmón a la macroscopía, y a la histología, menor número de alvéolos, ductos alveolares y alvéolos de mayor tamaño, lo que se asocia con un área de superficie alveolar menor (4). Lo anterior parece estar relacionado con una falla en la multiplicación alveolar del período postnatal, lo que no es observado en el último tiempo de gestación. También se ha descrito mayor frecuencia de doble red capilar en niños con SD (4).

Los quistes subpleurales están presentes en 20$36 \%$ de los niños con SD, siendo muy raros en niños sin SD. En general, no se detectan en una radiografía simple, sino en una tomografía computada de tórax. Los quistes subpleurales son numerosos, pequeños (1 a $4 \mathrm{~mm}$ de diámetro) y comunicados con el espacio aéreo proximal. En general, no tienen impacto clínico, siendo un hallazgo radiológico. Usualmente son de manejo conservador. Se debe tener en cuenta su presencia en caso de cirugía o uso de ventilación mecánica, por posibilidad de neumotórax $(3,4)$.

También pueden encontrarse enfermedades difusas del parénquima pulmonar manifestadas por alteraciones radiológicas crónicas, síntomas y signos respiratorios persistentes como disnea, tos, sibilancias, crepitaciones 0 hipoxia. Se debe estudiar como diagnóstico diferencial de enfermedad intersticial, descartando causas secundarias como complicaciones postinfecciosas, aspiración pulmonar, displasia broncopulmonar, hemosiderosis pulmonar, y enfermedad cardíaca y vascular pulmonar. (3).

\section{REFLUJO GASTRO-ESOFÁGICO Y ASPIRACIÓN PULMONAR}

El reflujo gastro-esofágico (RGE) es la condición que se produce al refluir contenido del estómago causando síntomas y/o complicaciones. Puede producir complicaciones respiratorias como aspiración pulmonar y neumonía aspirativa. Para su diagnóstico se requiere una historia compatible y exámenes como esofagograma y pHmetria esofágica. Según el caso, el tratamiento puede ser conservador, farmacológico o quirúrgico. $(4,19)$.

Los pacientes con SD tienen con mayor frecuencia trastornos de la deglución, y generalmente, aspiración pulmonar silenciosa, sin presentar tos ni ahogo. Un estudio describe 40\% de aspiración pulmonar silenciosa en niños con SD con síntomas respiratorios crónicos en los que se realizó videodeglución (18). La aspiración pulmonar crónica secundaria a trastorno de la deglución puede causar sibilancias persistentes, tos crónica, neumonía recurrente, daño pulmonar y/o deterioro de la función pulmonar. El examen de elección para el diagnóstico es la videofluoroscopía de deglución $(4,19)$.

\section{CONCLUSIONES}

Los niños con SD tienen predisposición para presentar una variedad de problemas respiratorios, los que muchas veces 
son subdiagnosticados. Los TRS son muy frecuentes, y se debe tener una alta sospecha para evitar mayores consecuencias conductuales, cognitivas y además la aparición de HTP. Los pacientes que presentan sibilancias recurrentes no siempre serán asmáticos; si no responden a tratamiento se debe realizar precozmente estudio de otras posibles causas como aspiración pulmonar 0 alteraciones de la vía aérea.

El conocimiento de todos los problemas respiratorios que pueden presentar los niños con SD es fundamental, para siempre tener la sospecha, realizar un adecuado diagnóstico y tratamiento, y con ello prevenir complicaciones, mejorar la calidad de vida y desarrollo físico e intelectual de estos niños.

Los autores declaran no presentar conflicto de interés.

\section{REFERENCIAS}

1. Weijerman ME, Winter JP. The care of children with Down syndrome. Eur J Pediatr 2010; 169:1445-1452

2. Lizama M, Retamales N, Mellado C. Recomendaciones de cuidados en salud de personas con síndrome de Down: 0 a 18 años. Rev Med Chile 2013; 141: 80-89

3. McDowell KM, Craven DI. Pulmonary Complications of Down Syndrome during Childhood. J Peds 2011; 158: 319-325

4. Watts R, Vyas H. An overview of respiratory problems in children with Down's syndrome. Arch Dis Child 2013; 98: 812-817

5. Retamales N, Moreno R, González A, Cerda J, Lizama M. Morbilidad y Mortalidad durante el Primer Año de Vida en Pacientes con Síndrome de Down. Rev Chil Pediatr 2009; 80: 323-331

6. Fitzgerald DA, Paul A, Richmond C. Severity of obstructive apnoea in children with Down syndrome who snore. Arch Dis Child 2007; 92: 423-5

7. Bull MJ and the Committee on Genetics Health Supervision for Children With Down Syndrome. Pediatr 2011;128: 393-406

8. Kuhle S, Urschitz MS, Eitner S, Poets CF. Interventions for obstructive sleep apnea in children: A systematic review. Sleep Med Rev 2009; 13: e123-e131

9. Holty JC, Guilleminault C. Surgical Options for the Treatment of Obstructive Sleep Apnea. Med Clin N Am 2010; 94: 479-515

10. Bertrand P, Navarro H, Caussade S, Holmgren N, Sánchez I. Airway Anomalies in Children With Down Syndrome: Endoscopic Findings. Pediatric Pulmonology 2003; 36: 137-141

11. Jacobs IN, Gray RF, Todd NW. Upper airway obstruction in children with Down syndrome. Arch Otolaryngol Head Neck Surg 1996;122: 945-50

12. Miller R, Gray SD, Cotton RT, Myer CM III, Netterville J. Subglottic stenosis and Down syndrome. Am J Otolaryngol 1990;11:274-7 13. Shott SR. Down syndrome: analysis of airway size and a guide for appropriate intubation. Laryngoscope 2000; 110: 585-92

14. Bloemers BLP, van Furth AM, Weijerman ME, Gemke RJ, Broers Ch, Kimpen JL, Bont L. High incidence of recurrent wheeze in children with Down syndrome with and without previous respiratory syncytial virus lower respiratory tract infection. Pediatr Infect Dis J 2010; 29:39-42

15. Hilton JM, Fitzgerald DA, Cooper DM. Respiratory morbidity of hospitalized children with Trisomy 21. J Paediatr Child Health 1999;35:383-6
16. Bloemers BL, van Furth AM, Weijerman ME, Gemke RJ, Broers ChJ, Kimpen JL, Bont L. Down syndrome: a novel risk factor for respiratory syncytial virus bronchiolitis-a prospective birth-cohort study. Pediatr 2007;120:e1076-e1081

17. Ram G, Chinen J. Infections and immunodeficiency in Down syndrome. Clin Exp Immunol 2011; 164: 9-16

18. Frazier JB, Friedman B. Swallow function in children with Down syndrome: a retrospective study. Dev Med Child Neurol 1996; 38: 695-703

19. Pandit Ch, Fitzgerald DA. Respiratory problems in children with Down syndrome. J Paediatr and Child Health 2012; 48: E147E152 